Rafael Fabiano Machado Rosa ${ }^{1}$

Raquel Papandreus Dibl $\left.\right|^{2}$

Jamille dos Santos Picetti ${ }^{3}$

Rosana Cardoso Manique Rosa ${ }^{4}$

Paulo Ricardo Gazzola ZeN ${ }^{5}$

Carla Graziadio ${ }^{6}$

GIORGIO AdRIANO PASKULIN ${ }^{7}$

\title{
Amenorréia e anormalidades do cromossomo $X$
}

\author{
Amenorrbea and X chromosome abnormalities
}

\author{
Palavras-chave \\ Amenorréia/genética \\ Cromossomo $X$ \\ Síndrome de Turner \\ Cariótipo
}

Keywords

Amenorrhea/genetics

X chromosome

Turner syndrome

Karyotype

Diagnostic techniques and procedures

\section{Resumo}

OBJETIVO: correlacionar as manifestações clínicas de pacientes com amenorréia e anormalidades do cromossomo X. MÉTODOS: realizou-se uma análise retrospectiva dos achados clínicos e laboratoriais das pacientes com amenorréia e anormalidades do cromossomo X, atendidas entre janeiro de 1975 e novembro de 2007. Suas medidas antropométricas foram avaliadas através de tabelas de crescimento padrão, sendo que, quando presentes, dismorfias menores e maiores foram anotadas. $\bigcirc$ estudo dos cromossomos foi realizado através do cariótipo com bandamento GTG. RESULTADOS: do total de 141 pacientes com amenorréia, 16\% apresentavam anormalidades numéricas e $13 \%$ estruturais do cromossomo X. Destas pacientes com anormalidade do $X(n=41), 35$ possuíam descrição clínica completa. Todas elas apresentavam hipogonadismo hipergonadotrófico. Amenorréia primária foi observada em 24 pacientes, das quais 91 ,7\% com fenótipo de síndrome de Turner. Com exceção de um caso com deleção Xq22-q28, todas as demais pacientes com este fenótipo apresentavam alterações envolvendo Xp (uma com uma linhagem 46,XY associada). Os dois casos restantes com apenas amenorréia primária possuíam deleções proximais de Xq. Entre as 11 pacientes com amenorréia secundária, 54,5\% apresentavam fenótipo de Turner (todas com monossomia do X isolada ou em mosaico). Entre aquelas com fenótipo de falência ovariana isolada observaram-se somente deleções Xq e trissomia do X. CONCLUSÕES: a análise cromossômica deve sempre ser realizada em mulheres com falência ovariana de causa não conhecida, mesmo na ausência de achados dismórficos. Esta também é de extrema importância em pacientes sindrômicas, pois, além de confirmar o diagnóstico, é capaz de identificar pacientes em risco, como nos casos com uma linhagem 46,XY.

\section{Abstract}

PURPOSE: to correlate the clinical manifestations of patients with amenorrhea and $X$ chromosome abnormalities. METHODS: a retrospective analysis of the clinical and laboratorial findings of patients with amenorrhea and abnormalities of X chromosome, attended between January 1975 and November 2007 was performed. Their anthropometric measures were evaluated through standard growth tables, and, when present, minor and major anomalies were noted. The chromosomal study was performed through the GTG banded karyotype. RESULTS: from the total of 141 patients with amenorrhea, $16 \%$ presented numerical and $13 \%$ structural abnormalities of $X$ chromosome. From these patients with $X$ chromosome abnormalities $(n=41), 35$ had a complete clinical description. All presented hypergonadotrophic hypogonadism. Primary amenorrhea was observed in 24 patients, $91.7 \%$ of them with a Turner syndrome phenotype.
Correspondência:

Giorgio Adriano Paskulin

Genética Clínica - Universidade Federal de Ciências da Saúde de

Porto Alegre

Avenida Sarmento Leite, 245, sala 403 - Centro CEP 90050-170 - Porto Alegre/RS Fone: (51) 3303-8771/Fax: (51) 3303-8810

E-mail: paskulin@ufcspa.edu.br

Recebido

$5 / 9 / 08$

Aceito com modificações $8 / 9 / 08$

\section{Complexo Hospitalar Santa Casa de Porto Alegre da Universidade Federal de Ciências da Saúde de Porto Alegre - UFCSPA - Porto} Alegre (RS), Brasil.

Geneticista Clínico da Universidade Federal de Ciências da Saúde de Porto Alegre - UFCSPA - do Complexo Hospitalar Santa Casa de Porto Alegre - CHSCPA - Porto Alegre (RS), Brasil; Pós-graduando do Programa de Pós-graduação em Patologia da Universidade Federal de Ciências da Saúde de Porto Alegre - UFCSPA - Porto Alegre (RS), Brasil.

2 Preceptora do Serviço de Ginecologia e Obstetrícia do Complexo Hospitalar Santa Casa de Porto Alegre - CHSCPA - Porto Alegre (RS), Brasil; Pós-graduanda do Programa de Pós-graduação em Patologia da Universidade Federal de Ciências da Saúde de Porto Alegre - UFCSPA - Porto Alegre (RS), Brasil.

${ }^{3}$ Acadêmica do Curso de Medicina da Universidade Federal de Ciências da Saúde de Porto Alegre - UFCSPA - Porto Alegre (RS), Brasil.

${ }^{4}$ Médica formada pela Universidade Federal de Ciências da Saúde de Porto Alegre - UFCSPA - Porto Alegre (RS), Brasil.

${ }^{5}$ Geneticista Clínico da Universidade Federal de Ciências da Saúde de Porto Alegre - UFCSPA - e do Complexo Hospitalar Santa Casa de Porto Alegre - CHSCPA - Porto Alegre (RS), Brasil; Professor Adjunto da Disciplina de Genética Clínica da Universidade Federal de Ciências da Saúde de Porto Alegre - UFCSPA - Porto Alegre (RS), Brasil.

${ }^{\circ}$ Geneticista Clínica da Universidade Federal de Ciências da Saúde de Porto Alegre - UFCSPA - e do Complexo Hospitalar Santa Casa de Porto Alegre - CHSCPA - Porto Alegre (RS), Brasil; Professora-assistente da Disciplina de Genética Clínica da Universidade Federal de Ciências da Saúde de Porto Alegre - UFCSPA - Porto Alegre (RS), Brasil.

Geneticista Clínico da Universidade Federal de Ciências da Saúde de Porto Alegre - UFCSPA - e do Complexo Hospitalar Santa Casa de Porto Alegre - CHSCPA - Porto Alegre (RS), Brasil; Citogeneticista Responsável pelo Laboratório de Citogenética da Universidade Federal de Ciências da Saúde de Porto Alegre - UFCSPA - Porto Alegre (RS), Brasil; Professor-Associado da Disciplina de Genética Clínica da Universidade Federal de Ciências da Saúde de Porto Alegre - UFCSPA - Porto Alegre (RS), Brasil. 
Despite a case with $X_{q 22-q 28}$ deletion, all patients with this phenotype presented alterations involving Xp (one case with an additional cell lineage $46, X Y$ ). The two remaining patients with only primary amenorrhea had proximal deletions of Xq. Among the 11 patients with secondary amenorrhea, $54.5 \%$ presented a Turner phenotype (all with isolated or mosaic X chromosome monosomy). Patients with phenotype of isolated ovarian failure had only Xq deletions and X trisomy. CONCLUSIONS: the cytogenetic analysis must always be performed in women with ovarian failure of unknown cause, even in the absence of clinical dysmorphic features. This analysis is also extremely relevant in syndromic patients, because it can either confirm the diagnosis or identify patients in risk, like the cases involving a 46,XY lineage.

\section{Introdução}

A amenorréia representa um sintoma, e não uma doença, e caracteriza-se pela falta (amenorréia primária) ou cessação anormal da menstruação (amenorréia secundária). Excluindo-se os casos de gestação, lactação e menopausa, tem sido descrita em cerca de 3 a $4 \%$ das mulheres, sendo decorrente tanto de disfunções endocrinológicas e anomalias gonadais, quanto de doenças genéticas específicas, como anormalidades cromossômicas ${ }^{1,2}$. Estas últimas começaram a ser identificadas a partir do final da década de 1950, com o desenvolvimento do exame de cariótipo, sendo que foram melhor caracterizadas a partir de 1970 com o surgimento das técnicas de bandamento ${ }^{3}$ e sincronização dos cromossomos ${ }^{4}$, e, mais recentemente, com a hibridização in situ fluorescente (FISH) ${ }^{5}$, uma técnica moderna que integra a citogenética clássica com a genética molecular. Atualmente, sabe-se que cerca de 16 a 63\% dos casos de amenorréia primária e 4 a $44 \%$ de secundária são de causa cromossômica, com especial incidência para as anormalidades envolvendo o cromossomo $\mathrm{X}^{6,7}$. Estas englobam alterações tanto numéricas quanto estruturais deste cromossomo. $\mathrm{O}$ cromossomo $\mathrm{X}$ apresenta um braço curto ( $\mathrm{p}$, do francês petit = pequeno) e, por convenção, um longo (q), separados por uma zona de constrição (o centrômero). As anormalidades numéricas caracterizam-se pela ausência (monossomia - 45,X) ou adição (trissomia - 47,XXX) de todo um cromossomo X. Por sua vez, as anormalidades estruturais incluem a ausência (deleção) de segmentos cromossômicos do braço curto [46,X, del(Xp)] e longo do cromossomo X [46,X, $\operatorname{del}(\mathrm{Xq})]$, a perda do seu braço curto (p) com duplicação do seu braço longo (q) [isocromossomo do braço longo do X - 46,X,i(Xq)] e a perda parcial de suas extremidades com fusão formando uma estrutura em forma de anel [cromossomo X em anel - 46,X,r(X)]. Estas anormalidades podem ocorrer tanto na forma isolada como em conjunto com outras linhagens celulares, constituindo o que se denomina de mosaicismo (mos). Estas alterações do X se caracterizam, clinicamente, por disgenesia gonadal com falência ovariana que leva, após o momento da puberdade, a um quadro de hipogonadismo hipergonadotrófico. Sabe-se hoje que existem genes localizados ao longo do cromossomo $\mathrm{X}$ que se relacionam tanto com a função gonadal como também com a presença de outros achados clínicos específicos, como a baixa estatura, que caracterizam a chamada síndrome de Ullrich-Turner (mais conhecida como síndrome de Turner) ${ }^{8-10}$. Apesar da significativa freqüência destas anormalidades cromossômicas entre pacientes com amenorréia, são raras as suas descrições em nosso meio ${ }^{9,11}$.

Assim, o objetivo do nosso trabalho foi verificar as características clínicas e citogenéticas de pacientes com amenorréia e anormalidades do cromossomo $\mathrm{X}$ avaliadas em nosso serviço.

\section{Métodos}

Este estudo incluiu todas as pacientes encaminhadas ao Serviço de Genética Clínica da Universidade Federal de Ciências da Saúde de Porto Alegre (UFCSPA), entre janeiro de 1975 e novembro de 2007, por apresentarem queixa de amenorréia. Todas apresentavam evidência, na sua análise citogenética, de uma alteração numérica e/ou estrutural do cromossomo X.

Realizou-se uma análise retrospectiva das suas características clínicas, laboratoriais e citogenéticas, com revisão sistemática de suas fichas de atendimento e preenchimento de um protocolo padrão. Medidas antropométricas (altura, peso e perímetro cefálico) foram avaliadas através de tabelas de crescimento padrão ${ }^{12}$, sendo que os valores que se encontravam dois desvios padrão acima ou abaixo da média foram considerados anormais. Quando presentes, dismorfias menores (anomalias anatômicas pequenas sem conseqüências médicas ou cosméticas importantes, como palato alto, cúbito valgo e unhas hiperconvexas) e maiores (alterações com implicação médica ou cosmética importante, como defeitos cardíacos congênitos e anomalias renais) foram anotadas de acordo com a terminologia adotada por Merks et al. ${ }^{13}$. Pacientes portadoras de alterações estruturais do cromossomo $\mathrm{X}$ nas quais a origem do fragmento cromossômico adicional não pôde ser elucidada ou que apresentavam prontuários com a descrição clínica incompleta foram excluídas da amostra.

O estudo dos cromossomos foi realizado no Laboratório de Citogenética da UFCSPA através do exame de cariótipo com bandamento GTG. Resumidamente, esta técnica inclui a preparação de cultivos celulares a partir de sangue periférico (para análise dos linfócitos), tratamento dos mesmos com colchicina, choque hipotônico, fixação das células e preparo das lâminas para posterior coloração por bandas G com tripsina e Giemsa (GTG). A avaliação das lâminas foi realizada em um microscópio Axioskop 
Zeiss (utilizando-se de uma objetiva com aumento de cem vezes com imersão a óleo), sendo que foram analisadas, em média, 35 metáfases para cada caso. Os resultados dos cariótipos foram avaliados de acordo com as normas presentes no International System for Human Cytogenetic Nomenclature (ISCN $)^{14}$, o sistema de nomenclatura padrão atualmente utilizado e mundialmente aceito para descrição das alterações numéricas e estruturais dos cromossomos. Este estudo foi aprovado pelo Comitê de Ética em Pesquisa da UFCSPA.

\section{Resultados}

Das 141 pacientes com amenorréia encaminhadas para avaliação genética, 101 apresentavam amenorréia primária e 40 secundária. Em todas elas o exame de cariótipo havia sido realizado com sucesso. Anormalidades citogenéticas envolvendo o cromossomo $\mathrm{X}$ foram observadas em 41 pacientes, sendo em $23(56 \%)$ alterações numéricas e em $18(44 \%)$ estruturais. Destas, seis pacientes foram excluídas da amostra ou por serem portadoras de uma alteração estrutural do cromossomo X, na qual o fragmento cromossômico adicional não pôde ser identificado $(\mathrm{n}=2)$, ou por apresentarem prontuários com a descrição clínica incompleta $(n=4)$. Assim, a amostra final foi constituída por 35 pacientes. Suas idades variaram de 15 a 47 anos, sendo em média de 21,7 anos para aquelas com amenorréia primária e 32,6 anos para aquelas com amenorréia secundária. Todas elas apresentavam um quadro hormonal de hipogonadismo hipergonadotrófico. Das pacientes com amenorréia primária $(n=24), 16$ $(66,7 \%)$ eram portadoras de alterações numéricas do cromossomo X e oito $(33,3 \%)$ estruturais. Vinte e duas $(91,7 \%)$ apresentavam fenótipo de síndrome de Turner, sendo que, com exceção de um caso de deleção parcial do braço longo do X envolvendo a região q22-q28, todas apresentavam alterações do braço curto do X (monossomia de todo cromossomo $\mathrm{X}$, deleção envolvendo somente o braço curto ou isocromossomo do braço longo do X). Os dois casos restantes, com queixa menstrual somente, apresentavam deleções envolvendo regiões proximais do braço longo do X (Tabela 1 e Figura 1).

Tabela 1 - Características clínicas e anormalidades citogenéticas do cromossomo X observadas nos pacientes da amostra ( $n=35$ )

\begin{tabular}{|c|c|c|c|c|c|c|c|c|c|c|c|c|}
\hline Alteração cromossômica* & $\begin{array}{l}x \\
\text { y }\end{array}$ & 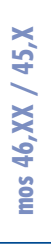 & 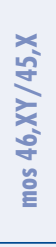 & 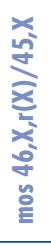 & 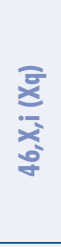 & 峁產 & 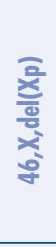 & 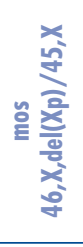 & 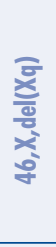 & $\begin{array}{l}\underset{x}{x} \\
\text { 爻 }\end{array}$ & 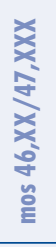 & 흥 \\
\hline Total de casos & 15 & 3 & 1 & 2 & 2 & 1 & 2 & 1 & 6 & 1 & 1 & 35 \\
\hline Baixa estatura $^{\varphi}$ & 13 & 2 & 1 & 2 & 2 & 1 & 2 & 1 & 1 & & & 25 \\
\hline Implantação anormal dos cabelos & 10 & & 1 & 1 & 1 & & 1 & 1 & 1 & & & 16 \\
\hline Cúbito valgo & 9 & 1 & 1 & 1 & 1 & & 2 & 1 & & & & 16 \\
\hline Unhas hiperconvexas/displásicas & 10 & & 1 & 1 & 1 & & 2 & & & & & 15 \\
\hline Tórax em escudo & 8 & & & 1 & 1 & & 2 & & 1 & & & 13 \\
\hline Palato alto & 4 & & & & & & 1 & & & & & 5 \\
\hline Encurtamento de metacarpianos $\left(+4^{\circ}\right)$ & 3 & & 1 & & & & & & & & & 4 \\
\hline Pregas epicânticas & 3 & & & & & 1 & & & & & & 4 \\
\hline Alterações oftalmológicas & 2 & & & & & 1 & & & & & & 3 \\
\hline Deficit auditivo & 2 & & & & & & 1 & & & & & 3 \\
\hline Alterações cardiológicas & 2 & & & 1 & & & & & & & & 3 \\
\hline Hipotireoidismo & 1 & 1 & & 1 & & & & & & & & 3 \\
\hline Otites de repetição & 1 & & 1 & & & & & & & & & 2 \\
\hline Micrognatia & 2 & & & & & & & & & & & 2 \\
\hline Cifoescoliose & 1 & & & & & 1 & & & & & & 2 \\
\hline
\end{tabular}




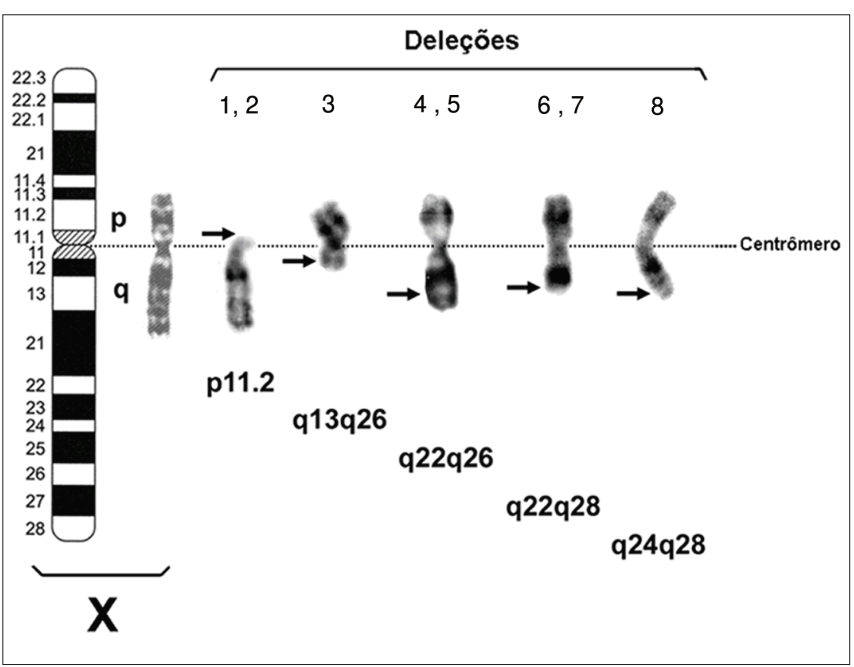

Figura 1 - Ideograma e ilustração de um cromossomo $X$ normal $(X)$, juntamente com as imagens dos casos de deleção do $X$ presentes em nossa amostra, que envolviam os segmentos pl1.2-pter do braço curto (1 e 2) e as regiões q13-q26 (3), q22-q26 (4 e 5), q22-q28 16 e 7) e q24-q28 (8) do seu braço longo (as setas indicam os respectivos pontos onde ocorreram as deleções). $\bigcirc$ fenótipo destas pacientes foi de amenorréia primária $\operatorname{com}(1,2$ e 6$)$ e sem (4 e 5) estigmas da síndrome de Turner, e de somente amenorréia secundária $(3,7$ e 8$)$

Das pacientes com amenorréia secundária $(\mathrm{n}=11)$, cinco $(45,5 \%)$ apresentavam alterações numéricas do cromossomo $\mathrm{X}$ e seis $(54,5 \%)$ estruturais. A média de idade da menarca destas pacientes variou de nove a 15 anos (média de 12,3 anos) e do início da falência ovariana, de 15 a 20 anos (média de 17,1 anos). As sete pacientes com fenótipo de síndrome de Turner $(54,5 \%)$ tinham, todas, monossomia do cromossomo $\mathrm{X}$ isolada ou em mosaico. Nas pacientes com fenótipo apenas de falência ovariana precoce $(45,5 \%)$, observaram-se somente deleções envolvendo as regiões distais do braço longo do $\mathrm{X}(\mathrm{n}=3)$ e trissomia do cromossomo $\mathrm{X}$ na forma isolada ou em mosaico $(\mathrm{n}=2)$, vide Tabela 1 e Figura 1.

A média da estatura das pacientes com constituição cromossômica 45,X do total da amostra foi de $141 \mathrm{~cm}$ (igual à daquelas com isocromossomo do braço longo do $\mathrm{X}$ ), e dos mosaicos (envolvendo uma linhagem $45, \mathrm{X})$ foi de $145 \mathrm{~cm}$, igual à observada em pacientes com deleção do braço curto do X. Comparando-se os achados clínicos das pacientes $45, \mathrm{X}$ com aquelas em mosaico, observou-se que as primeiras apresentaram, mais freqüentemente, um quadro de amenorréia primária associada à baixa estatura e dismorfias tanto menores como maiores. Em três pacientes da amostra (todas com alterações citogenéticas envolvendo uma linhagem 45, $\mathrm{X}$ isolada ou em mosaico) verificou-se também hipotireoidismo (Tabela 1).

\section{Discussão}

$\mathrm{Na}$ ausência de um cromossomo $\mathrm{Y}$, a gônada indiferenciada desenvolve-se como um ovário. Contudo, para que ocorra o seu adequado funcionamento é imprescindível que ambos os cromossomos $\mathrm{X}$ presentes estejam íntegros. A associação de anormalidades neste cromossomo com o desenvolvimento de falência ovariana confirma esta observação. Nestes casos, a gônada normal acaba sendo trocada por uma estrutura branca e fibrosa em forma de fita, localizada na posição originalmente ocupada pelo ovário ${ }^{8,15,16}$. Atualmente, sabe-se que algumas regiões do cromossomo têm sido relacionadas à falência ovariana: a falência ovariana prematura um (FOP1), que abrange a região Xq26qter, e a falência ovariana prematura dois (FOP2), em

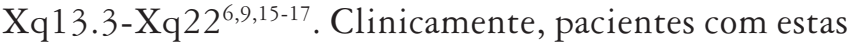
anormalidades apresentam um quadro de amenorréia primária com infantilismo sexual, ou de amenorréia secundária acompanhada de esterilidade ${ }^{15}$. Além disso, estes indivíduos mostram, muito comumente, achados clínicos adicionais que caracterizam a síndrome de Turner. Isto se deve ao envolvimento de outros genes presentes no cromossomo X, em especial no seu braço curto (p), como o SHOX (short stature homebox containing gene), que se associa tanto com a baixa estatura como com certas alterações esqueléticas, tais como o palato ogival, quarto metacarpiano curto, cúbito valgo e deformidade de Madelung ${ }^{18}$. Atualmente, sabe-se que as anormalidades citogenéticas do cromossomo $\mathrm{X}$ são freqüentes entre pacientes com amenorréia, especialmente do tipo primária, sendo que profissionais da área da saúde, em especial Ginecologistas e Endocrinologistas, devem estar cientes da importância deste diagnóstico, tanto para avaliação como para manejo destes indivíduos.

A anormalidade do cromossomo $\mathrm{X}$, mais freqüentemente observada entre pacientes com amenorréia, é a monossomia do X $(45, \mathrm{X})$. Esta representa também a principal constituição cromossômica de pacientes com síndrome de Turner (cerca de metade dos casos) ${ }^{16,19,20}$, tendo sido observada em $42,9 \%$ das pacientes de nossa amostra. Estas apresentaram de uma forma geral, um quadro clínico com estigmas da síndrome de Turner, como baixa estatura, associados, algumas vezes, a anormalidades maiores, como cardiopatia congênita e malformação renal. Quanto ao perfil hormonal, a maioria possuía um quadro de amenorréia primária $(87 \%)$. Todas estas características clínicas foram concordantes com as descrições prévias feitas na literatura ${ }^{6,8,10,21}$.

A forma mais freqüente de mosaicismo associada à disgenesia gonadal envolve uma linhagem com 
monossomia do cromossomo $\mathrm{X}$ e outra feminina normal $(\operatorname{mos} 45, \mathrm{X} / 46, \mathrm{XX})^{8,16,19-21}$. Este é observado em aproximadamente 10 a $15 \%$ dos casos de síndrome de Turner, e representou a forma mais comum de mosaicismo de nosso estudo. Indivíduos com esta constituição cromossômica apresentam, tal como verificado nas três pacientes de nossa amostra, um número inferior de dismorfias menores, uma estatura média final maior e mais comumente amenorréia do tipo secundária do que pacientes com monossomia do $\mathrm{X}$ somente $(45, \mathrm{X})$. Anormalidades maiores, como alterações cardiológicas e renais, também são menos freqüentes nestas pacientes com mosaicismo, sendo que nenhuma das pacientes do nosso estudo as apresentava. Isto talvez tenha relação com o fato de estes indivíduos apresentarem uma constituição cromossômica com uma linhagem adicional de células normais $(46, \mathrm{XX})^{8}$.

Por sua vez, indivíduos com mosaicismo entre uma linhagem com monossomia do cromossomo $\mathrm{X}$ e outra masculina normal $(\operatorname{mos} 45, \mathrm{X} / 46, \mathrm{XY})$ podem ter gônadas em fita bilateralmente. Entretanto, mais freqüentemente, têm uma gônada em fita unilateral e um testículo disgenético contralateral associados a uma genitália externa ambígua ${ }^{8}$. Estas pacientes são descritas em cerca de 2 a $5 \%$ dos casos de síndrome de Turner. A importância de reconhecê-las reside no fato de que elas, pela presença de um cromossomo $\mathrm{Y}$, possuem um risco aumentado de malignização gonadal. Este risco aumenta com a idade, sendo estimado em $2 \%$ aos dez anos e $27 \%$ aos $30 \operatorname{anos}^{19,20}$. A única paciente de nossa amostra com esta constituiçãa cromossômica não possuía uma genitália ambígua e foi identificada somente após avaliação cariotípica. Neste caso, esta análise foi essencial para a indicação da gonadectomia profilática que acabou sendo realizada nesta paciente.

Anormalidades estruturais do cromossomo X, por outro lado, são variáveis e menos freqüentes em pacientes com amenorréia. Elas estão presentes em cerca de 10 a $20 \%$ dos indivíduos com síndrome de Turner ${ }^{19,20}$. Em nosso estudo, foram observadas em $34,3 \%$ das pacientes, e incluíram o isocromossomo do braço longo do $\mathrm{X}$ [46,X,i $(\mathrm{Xq})$ ], o cromossomo $\mathrm{X}$ em anel [46,X,r(X)] e deleções do braço curto $[46, \mathrm{X}, \operatorname{del}(\mathrm{Xp})]$ e longo do $\mathrm{X}[46, \mathrm{X}, \mathrm{del}(\mathrm{Xq})]$. Pacientes com isocromossomo do braço longo do X quase sempre apresentam um quadro com gônadas em fita, amenorréia primária e baixa estatura. Sua altura média final parece ser menor do que a descrita em indivíduos 45,X. Estes indivíduos têm também uma frequiência de anomalias maiores, similar à observada em indivíduos $45, \mathrm{X}^{8}$. Os casos de nossa amostra com isocromossomo do $\mathrm{X}(5,7 \%)$ caracterizaram-se por um quadro de amenorréia primária com achados típicos da síndrome de Turner sem a presença de anormalidades maiores. A sua estatura média foi igual à das pacientes $45, \mathrm{X}$, e nenhuma delas apresentou hipotireoidismo, um achado que, segundo a literatura, parece ser mais freqüente entre estes indivíduos (estes pacientes apresentariam uma predisposição maior para certos distúrbios auto-imunes, como a tireoidite de Hashimoto $^{19,21}$. O único caso de isocromossomo do braço longo do cromossomo $\mathrm{X}$ em mosaico de nossa amostra, que envolvia uma linhagem $45, \mathrm{X}$, apresentou amenorréia secundária e alterações fenotípicas da síndrome de Turner.

$\mathrm{O}$ cromossomo $\mathrm{X}$ em anel pode variar em tamanho e freqüentemente se associa a um quadro clínico atípico, ou mesmo mais severo de síndrome de Turner. Acreditase que isto ocorra porque, como proposto por certos autores, algumas destas pacientes apresentariam anéis com alteração do gene XIST (localizado na região q13.2 do cromossomo X), que é responsável pelo controle da inativação deste cromossomo ${ }^{7,8,10,19}$. Isto faria com que os cromossomos $\mathrm{X}$ em anel com alteração deste gene não se inativassem, levando a uma anormalidade pela maior expressão do seu material ${ }^{19}$. Em nossa amostra, encontramos apenas dois casos apresentando uma linhagem com cromossomo X em anel, ambos em mosaico. Contudo, estes se caracterizaram, fenotipicamente, por achados típicos da síndrome de Turner. Talvez isto tenha ocorrido pelo fato de os mesmos apresentarem um mosaicismo envolvendo uma linhagem $45, \mathrm{X}$, ou porque os cromossomos em anel em questão não envolviam o gene XIST.

Deleções do cromossomo X apresentam fenótipos variáveis, dependentes diretamente da região acometida neste cromossomo. Pacientes com deleções do braço curto $[46, \mathrm{X}, \operatorname{del}(\mathrm{Xp})]$, tanto isoladas como em mosaico com uma linhagem $45, \mathrm{X}$, geralmente apresentam um quadro de disgenesia gonadal com estigmas da síndrome de Turner ${ }^{8}$, tal como ocorreu nos três casos de nossa amostra. Em contraste, pacientes com deleções do braço longo do cromossomo X $[46, \mathrm{X}, \operatorname{del}(\mathrm{Xq})]$ dificilmente apresentam baixa estatura ou mesmo outros achados da síndrome de Turner ${ }^{9}$. Grandes deleções com pontos de quebra próximos à região q13 do cromossomo $\mathrm{X}$ (afetando mais FOP1) relacionam-se, na maioria das vezes, a um quadro de amenorréia primária de início entre 16 e 21 anos. Por outro lado, deleções mais terminais (acometendo mais FOP2) caracterizam-se por um quadro de falência ovariana precoce, de início principalmente entre 24 e 29 anos, e infertilidade ${ }^{8,9,15,16}$. Em nosso trabalho, não foi possível, devido ao número reduzido de casos e à diversidade das anormalidades cromossômicas encontradas, correlacionar as regiões de deleção do braço longo do cromossomo X com as 
características clínicas apresentadas pelas pacientes. Contudo, chamou-se a atenção ao fato de que, dos seis casos com deleção do braço longo do X, somente um (envolvendo a região q22-q28) apresentou achados da síndrome de Turner. Esta observação é concordante com a literatura, pois os principais genes conhecidos e relacionados ao fenótipo desta síndrome, como o SHOX, encontram-se presentes no braço curto do cromossomo $\mathrm{X}^{8}$.

Pacientes com trissomia do X $(47, \mathrm{XXX})$ também têm sido constantemente descritas com um quadro isolado de falência ovariana precoce $^{12}$, sendo que o primeiro relato desta associação foi feito em $1959^{16}$. A trissomia do $\mathrm{X}$ tem sido descrita em aproximadamente $4 \%$ das mulheres normais com falência ovariana precoce, sendo que anormalidades auto-imunes, como doenças da tireóide, parecem ser freqüentes nestas pacientes ${ }^{7}$. Outras alterações também muitas vezes associadas incluem alta estatura, deficit cognitivo, problemas de linguagem e alterações comportamentais ${ }^{12}$. As duas pacientes de nossa amostra, uma com trissomia do cromossomo $\mathrm{X}$ isolada e a outra em mosaico com uma linhagem celular normal $(46, \mathrm{XX})$, apresentaram um quadro exclusivamente de amenorréia secundária. Contudo, cabe lembrar que, frente ao desconhecimento da verdadeira freqüência destes indivíduos na população em geral (que se acredita ser alta), certos autores questionam se a relação entre a trissomia do cromossomo X e a falência ovariana pode ser, talvez, meramente ao acaso ${ }^{6}$.

No passado, a indicação da realização do exame de cariótipo era preferencialmente feita a pacientes com falência ovariana precoce com idade inferior a 30 anos $^{16}$. De forma interessante, em nosso estudo a idade mais elevada de falência ovariana foi 20 anos. Entretanto, estudos recentes têm mostrado que esta idade não pode ser considerada mais como limite, pois, apesar de não freqüentes, descrições de pacientes com alterações cromossômicas com idade de falência ovariana superior aos 30 anos têm sido realizadas ${ }^{16}$.

Assim, concluímos que a análise citogenética deve ser sempre realizada em mulheres com falência ovariana de causa não conhecida, mesmo na ausência de achados clínicos dismórficos sugestivos de alguma anormalidade cromossômica, pois, como verificado em nosso estudo, pacientes com deleções do braço longo do cromossomo X e portadoras de trissomia do $\mathrm{X}$, freqüentemente, apresentam a alteração menstrual como seu único achado clínico. Por outro lado, esta análise é de extrema importância mesmo em pacientes sindrômicas, pois é capaz, além de confirmar o diagnóstico, de identificar pacientes em risco, como aqueles casos envolvendo uma linhagem 46,XY.

\section{Referências}

1. Master-Hunter T, Heiman DL. Amenorrhea: evaluation and treatment. Am Fam Physician. 2006;73(8):1374-82.

2. Practice Committee of the American Society for Reproductive Medicine. Current evaluation of amenorrhea. Fertil Steril. 2006;8615 Suppl):S148-55.

3. Caspersson T, Zech L, Johansson C. Differential binding of alkylating fluorochromes in human chromosomes. Exp Cell Res. 1970;60(3):315-9.

4. Yunis JJ. New chromosome techniques in the study of human neoplasia. Hum Pathol. 1981;12(6):540-9.

5. Jauch A, Daumer C, Lichter P, Murken J, Schroeder-Kurth T, Cremer T. Chromosomal in situ suppression hybridization of human gonosomes and autosomes and its use in clinical cytogenetics. Hum Genet. 1990;85(2): 145-50.

6. Wong MS, Lam ST. Cytogenetic analysis of patients with primary and secondary amenorrhoea in Hong Kong: retrospective study. Hong Kong Med J. 2005; 11 (4):267-72.

7. Chrisoulidou A, Bili H, Georgiou E, Mavroudi S, Lazaridou AS. Mosaic ring $X$ chromosome in a case of secondary amenorrhea. Fertil Steril. In press 2008.

8. Simpson JL, Rajkovic A. Ovarian differentiation and gonadal failure. Am J Med Genet. 1999;89(4):186-200.
9. Badalotti M, Arent A, Polanczick A, Petracco R, Petracco A. Falência ovariana precoce associada a deleção no braço longo do cromossomo: relato de dois casos e revisão da literatura. Rev Bras Ginecol Obstet. 2006;28(9):551-6.

10. Monnier-Barbarino $P$, Forges $T$. Les bases génétiques de la défaillance ovarienne prématurée, J Gynecol Obstet Biol Reprod (Paris). 2002;31(4):333-42

11. Multani AS, Shah VC, Singh D, Chakravarty N, Chinoy NJ, Pathak S. Deletion/inversion in the $\mathrm{X}$-chromosome and increased telomeric associations in a female with primary amenorrhea. Braz J Genet. 1997;20(3):511-6.

12. Jones KL. Smith's recognizable patterns of human malformation. 6th edition. Philadelphia: Elsevier Saunders; 2006.

13. Merks JH, van Karnebeek CD, Caron HN, Hennekam RC. Phenotypic abnormalities: terminology and classification. Am J Med Genet A. 2003; 123A(3):211-30.

14. Shaffer LG, Tommerup N, editors. ISCN 2005. An International System for Human Cytogenetic Nomenclature. Basel: S.Karger; 2004.

15. Hassum Filho PA, Silva IDC, Vereschi ITN. O espectro das falências ovarianas ligadas ao cromossomo X. Arq Bras Endocrinol Metab. $2001 ; 45(4): 339-42$. 
16. Vilodre LC, Moretto M, Kohek MBF, Spritzer PM. Falência ovariana prematura: aspectos atuais. Arq Bras Endocrinol Metab. 2007;51(6):920-9.

17. Goswami D, Conway GS. Premature ovarian failure. Horm Res. 2007;68(4): 196-202.

18. Martins RRS, Ramos HIB, Llerena Júnior JC, Almeida JCC. Investigação clínica e genética em meninas com baixa estatura idiopática. Arq Bras Endocrinol Metab. 2003;47(6):684-94.
19. Cassidy SB, Allanson JE. Management of genetic syndromes. New York: Wiley-Liss; 2005.

20. Bufalino G, Fabrega R, González C, Romero G. Mosaicismo tuner 45XO/XY: diagnóstico diferencial con disgenesia gonadal mixta. Rev Obstet Ginecol Venezuela. 2007;67(1):61-6.

21. Gravholt $\mathrm{CH}$. Epidemiological, endocrine and metabolic features in Turner syndrome. Arq Bras Endocrinol Metabol. $2005 ; 49(1): 145-56$. 